\title{
Solving the Challenges of Pervasive Computing
}

\author{
Syed Muqsit Shaheed ${ }^{1}$, Jalil Abbas ${ }^{2}$, Asif Shabbir ${ }^{3}$, Fayyaz Khalid ${ }^{3}$ \\ ${ }^{1}$ Department of Computer Science \& IT, University of Lahore, Lahore, Pakistan \\ ${ }^{2}$ Department of Computer Science\& IT, Government Collage University Faisalabad (Layyah Campus), Layyah, \\ Pakistan \\ ${ }^{3}$ Department of Computer Science \& IT, University of Gujrat, Gujrat, Pakistan \\ Email: syedmuqsit@hotmail.com, ${ }^{*}$ sjshah786@gmail.com, rabtabox4u@gmail.com, fayyazrao@yahoo.com
}

Received 6 August 2015; accepted 12 September 2015; published 15 September 2015

Copyright (C) 2015 by authors and Scientific Research Publishing Inc.

This work is licensed under the Creative Commons Attribution International License (CC BY). http://creativecommons.org/licenses/by/4.0/

(c) () 0 pen Access

\begin{abstract}
Modern research emphasizes Pervasive Computing change faces, learning cultures, structures, communications, intellectual properties, information securities, data presentations and web displays to make attraction for human interaction. Pervasive systems have a broad range of applications but it is relatively challenging for pervasive applications to meet emergence into existing physical environment and newly built structure requirements. Due to their interaction to gather information and change the environment via activating devices independently is highlighted. Security of the pervasive devices and applications which control our activities has primary importance and will be destroyed, if the pervasive system operations are not secure. There is a need to improve the security measures for data to travel rapidly, unbroken, unchanged and invisible by deceptive recipients. Pervasive Computing allows users to get information and services access anytime and anywhere but need to discuss issues and solutions to deliver secure information with privacy and trust. Possible solutions for these challenges of Pervasive Computing interaction between human are emphasized. A collection of papers and articles have been collected in order to investigate the previous study of Pervasive Computing interaction and its challenges. Is it possible for us to understand what the scientific world will be close to generate new avenues? Expectations of future bring new openings for user interaction with systems, data, information and the environments in which they live, work and play.
\end{abstract}

\section{Keywords}

Pervasive Computing, Interaction, Human Computer Interaction, Challenges, Security, Trust, Privacy

"Corresponding author. 


\section{Introduction}

Human social network plays a remarkable role in the information spread in Cyber World. Pervasive systems refer to construct a universal computing environment where unified and invisible access to computing resources is provided to the user. Pervasive Computing interaction systems are rapidly discovering its way into every facet of our lives and change the aspects faster than speed. Large scale of interactive media facades challenges to emergence into existing physical environment and newly built structures, which need to achieve managing capabilities of varied stakeholders, existing work practices and schedules. This technology of Pervasive Computing is inter woven in human lives and due to this it has become the need of time. It aims to make our lives modest through the use of machine interaction by human [1] [2].

Innovation has changed our lives to an extensive degree and still can possibly transform it in another and emotional way. Due multiplication of innovation into our lives to an expansive degree we spend our lives in an alternate manner when contrasts with our forefathers. Innovation has connected into our everyday life exercises from attempting to recreational exercises. We are presently engaged in interaction with distinctive devices, gadgets and machines. Indeed, it interrupts into our life in such a route, to the point that it is exceptionally hard to recognize it separated from our surroundings [1].

Pervasive Computing is an energizing ideal model taking dynamic part in every domain of day by day exercises of human life, in every area and connection. The spirit of this thinking ahead of Pervasive Computing was initially depicted by Mark Weiser in 1991 in his research paper as; "The most profound technologies are those that disappear. They weave themselves into the fabric of everyday life, until they are in distinguish able from it” [2].

Mark Weiser is really an inspiration to consider PCs beyond the desktop model. Pervasive Computing has numerous areas for understandings and executions. It additionally alludes to technologies. Calm innovations will retreat into foundation of our everyday life exercises. This ideal model proposes that computing assets will show in such a way, to the point that client will not be mindful of their vicinity. An alternate part of calm processing is that these embedded devices give services little interaction with users so users don't occupy from their job in an innovation overpowering environment [3].

$\mathrm{HCI}$ is utilized to recognize and focus on the lively advancement of requirements and understandings spoke to at different phases of a utilization circumstance. The terminology calm processing, invisible processing, embedded interaction and unseen computers do not infer the size of the systems or consistent joining of the equipment into the client environment. These terms tend to depict the piece of communication and interaction with the computers. Keeping in mind the end goal to make the computer invisible to the user, it implies the interaction of the user with the computer is in a consistent manner join together with the user's tasks. User's focus is on the task instead of explicit interaction with the computer relationship where center is on computer instead of tasks. Interaction in the middle of human and machine brings consideration regarding, particular explanatory parts of interaction design are availability, interpretability and connectivity [4].

In this advanced time, this forefront innovation needs to meet distinctive interaction challenges and it is grouped by: attention, complexity, trust, security, privacy, usability and emergence. Pervasive Computing contains other different challenges like: assessment systems for Pervasive Computing applications, development cycle issues, social collaboration, user interface and engagement, emerging issues, cost and limitations of hardware and software. Furthermore, it was estimated by some researchers that the true value of Pervasive Computing interaction would come from the arrangement of the various computational components into a stronger and adaptable system than had formerly been possible [5] [6]. Security and privacy and trust are a standout amongst the most critical and challenging issues on Pervasive Computing. The way of the pervasive environment permits communications and devices pass through directly, whenever and anyplace, so current processing systems have ended up progressively pervasive. At the point when administrations are given effortlessly to all different networks and their users, clearly the real concern of users due to their critical data turns into a central point [7]. In this paper the security challenges over the applications created on Pervasive Computing environment and some security plans have been introduced.

The principal goal of the Pervasive Computing interaction is to permit user to attention on their everyday task instead of innovation. In last few years we watch numerous applications that accumulate user setting through sensors so as to do right things at correct time. HCI ought to consider usability as well as focus on helping user tasks, demonstrating access to data in most ideal way and focus on all the more influential manifestation of 
communications [1]. Pervasive Computing systems have utilization sensing, computing and correspondence abilities to watch and react to natural phenomena. Such system will inevitably empower computers to consistently incorporate into ordinary life. They have numerous potential applications in the workplace, home, health awareness, gaming, ecological checking and open transportation [8].

Follow as under, we discuss different requirements of Pervasive Computing interaction in section two. Pervasive system interaction solutions are described in section three. Security challenges of Pervasive Computing are also examined in section four. In section five we describe solution regarding security of pervasive systems. At the end of the paper conclusion of our study is given.

\section{Pervasive Interaction Requirements}

Pervasive applications interfaces ought to be versatile to their surroundings and act as indicated by the situation. They should adapt as per context and don't need explicit interaction from the user. The primary destination of Pervasive Computing is to permit user to emphasize on their task instead on technology. In few previous years we watch numerous applications that accumulate user context through sensors keeping in mind the end goal to do exact things at exact time. Applications most significant among these are Google Ad Sense, Ubi Cicero and Cyber Guide [9]. HCI should not only consider usability as well as focus on helping user tasks, demonstrating access to data in most ideal way and focus on all the more capable type of interaction. In the wake of thinking of its viewpoint HCI has taken new measurement particularly in Pervasive Computing. All the dream and curiosity of pervasive systems focused around the novel method for interaction with the systems. Some important appearances of Human Computer Interaction in Pervasive Computing are given below [1] [10] [11].

\subsection{Implicit Human Computer Interaction}

Traditional user interfaces were functioned oriented; the user got to whatever the system could do by indicating functions first and afterward their arguments [12]. The central point of IHCI is to emphasize on the user task by preference than stress on technology. IHCI in Pervasive Computing would be hidden and verifiable instead of conventional explicit interaction of user through the system. Because of powerful utilization of certain type of interaction the calm or hidden computers would be reality in not all but near future. The essential reason for calm and hidden computer is that system should to proactive as opposed to user include in explicit interaction with the system. Calm computing expels user from the system and trust that framework work positively as differed to the responsive user.

\subsection{Task Oriented Interaction}

Pervasive system environment ought to permit the user to focus on the task as opposed to on the technology. Pervasive systems permit the combination of and interaction between task and affective information processing. Pervasive devices with high level of tasks orientation are, consequently, a most significant objective.

\subsection{Multimodal of Interaction}

Multimodal communication furnishes the user with various modes of interfacing with a framework. A multimodal interface gives a few different tools to enter and yield of information. HCI in Pervasive Computing includes versatile to circumstance and backing for multimodal of interaction with the user. This requires an insights work coordinated with an ideal interaction model.

\subsection{Human Interaction Pattern}

Human interaction design in pervasive environment would multimodal of interaction reinforcing with the reliable interface, expressive interface, useful interface, ordered and suitable information.

\subsection{Natural Human Computer Interaction}

Human-computer collaboration has a tendency to rapid change, and advance to something users are used to, which is natural for them, not something they need to learn [13]. Natural Human-Computer Interaction (NHCI) aims to research new intuitive computing system that emerges from human language and behaviour into tech 
applications. NHCI supports interaction with computerized systems without the requirement of external equipment like mice or keyboards. Rather, these will focus on computing systems easy to use and replaced by speech recognitions, context awareness, gestures and body movement. With development in innovation the interaction in Pervasive Computing has an expansion to be carried with natural interactions.

\section{Solutions for Pervasive Systems Interaction}

Human computer interaction plays an important role in field of Pervasive Computing environment. There are some interaction solutions to face pervasive systems are proposed.

\subsection{Context Aware Interaction}

Devices in the natural world require being context aware in order to perform most excellent. Context-awareness is an energizing and testing zone of human and computer interaction. The fundamental thought is to give computers perceptual qualities ("eyes and ears") so as to make them perceive the circumstances in which user connect with data information system. Utilizing sensors, situation can be discovered and delegated contexts. Once the system has perceived in which context an interaction happens, this data can be utilized to change, trigger, and adjust the conduct of applications and systems. The input side of implicit human computer interaction takes a gander look at information that users produce with a specific end goal to connect with this present reality and along these lines gives a speculation of context-awareness in human computer interaction.

A design context-aware interactive system is difficult. One needs to remember that user figure out how to communicate with systems, and that they adjust their behavior. It is vital that must be familiar with the changing and adaptive behavior of the application and connect it to the situations they are in. Else, they will have an exceptionally troublesome time figuring out how to utilize the system. Well-made designed context-awareness is an eminent and dominant way to develop user-friendly and pleasurable applications [14]. These days we carry on a huge upgrade in specialized tools of communication and here are numerous methods that can be utilized by individuals to impart, in the same way as instant messaging, email or telephone. This upgrade carries a definite stress measure on individuals, particularly for workers, on the grounds that they are losing control on how and when they can be arrived at. One of the conceivable answers to reduce this measure of anxiety is to assign the power of interferences to the user's contact by distributed his contextual data. Distributed information will facilitate his contacts to assess the effect of interference by initiating created an interaction with him [15]. From this thought B. Chihaniinfer a model which was called HEP (en Hanced unint Erru Ption) [15] [16] with the mean to suggest the best specialized apparatus in view of the callee setting.

Figure 1 shows the structural application design of the mobile. The HEP Application (Figure 2) is an Android based application improving user's contacts address book with user location, contextual information and activity.

After the obtained context we make user's workload status that is then showed to the user's look closely in order to notify them about his availability to reply a call or look at message received [15]. Figure 3 shows possible workloads in different statuses.

\subsection{Multimodal, Natural and Intelligent Interfaces}

A different independent single channel is called a modality. The information transferred between user and system through single channel. Single channel based system called uni-model and system based with multiple channels is called multi-model [1]. A multimodal interface refers to the pattern dependent on multiple modalities. The description of these interfaces is acquired from human sorts of interaction which are essentially his abilities: hearing, sight, touch, taste and smell. The potential outcome for interaction with a machine involves however types are not bound to these. Accordingly, a multimodal interface work as an organizer of human-PC communication by means of two or more types of information that go past the conventional mouse and console. The accurate number of supported input modes, their types and the method in which they perform task collectively may shift broadly starting with one multimodal framework then onto the next. Multimodal interfaces join various models of facial expressions, speech, gaze, motion and other non-customary types of input. A standout amongst the most generally provided pattern of input strategies is that of speech and gesture. A fascinating part of multimodality is the cooperation of several types of modalities to support the recognition. Case in point, visu- 


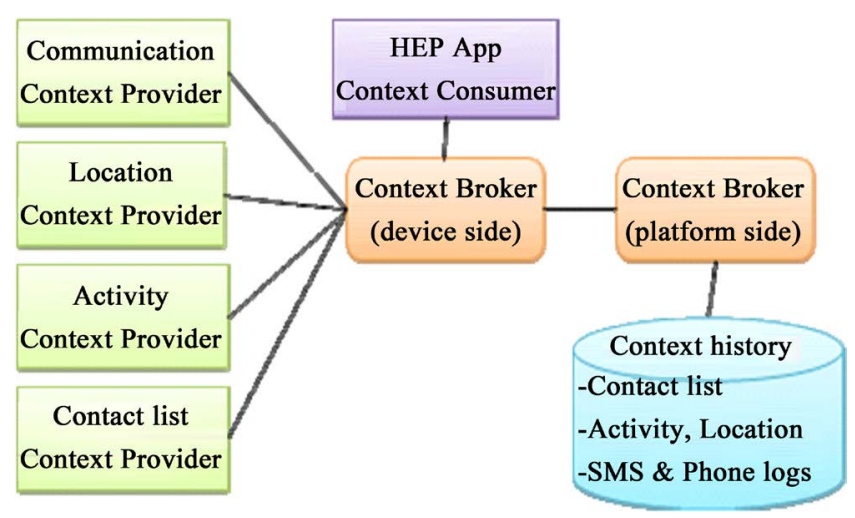

Figure 1. HEP architecture [15].

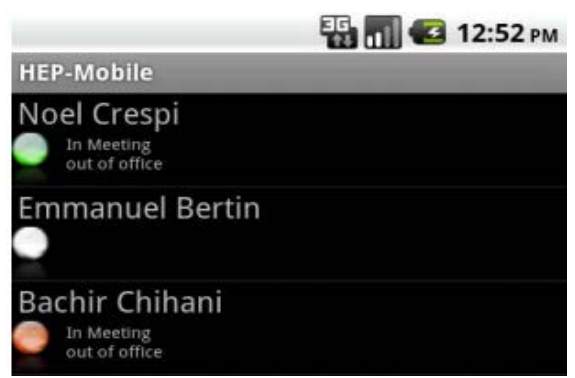

Figure 2. a snapshot from HEP Android Application [15].

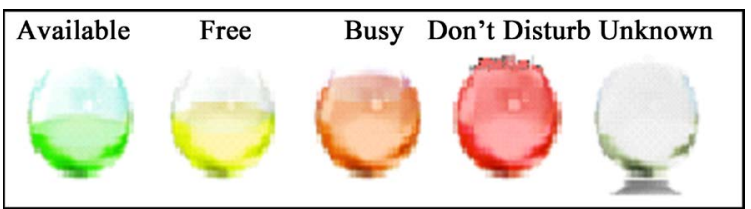

Figure 3. Workload status [15].

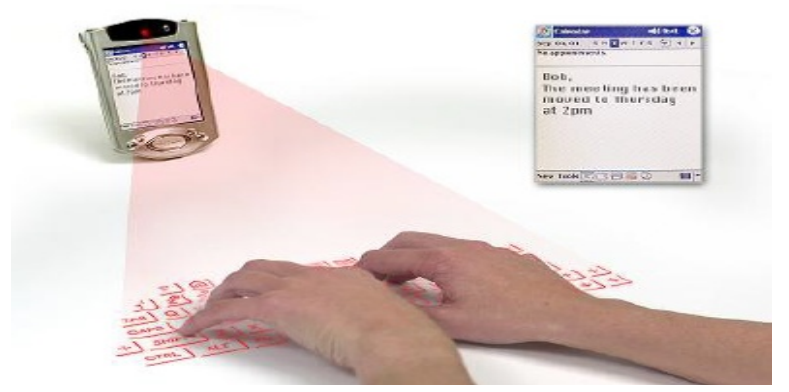

Figure 4. Canesta key board [18].

al-based tracking of lip movement can support sound based speech recognition methods and these methods of recognition can support command securing in visual-based recognition of gesture [17]. Study as case in example, here is the answer for keyboarding that has been suggested by "Compaq's iPAQ" which is called "Canesta keyboard" as demonstrated in Figure 4. It is a virtual console that is anticipating a "QWERTY" as example on a hard surface by utilizing a red light. At that point gadget tries to track movement of user's finger though writing at first glance with a motion sensor and transmit the keystrokes once more to the machine [17] [18].

Smart user interfaces mean to improve the productivity, adequacy and naturalness of HCI by utilizing the techniques of artificial intelligence. If we research history, user write down abilities starts with typewriter and typewriter change to keyboard, keyboard move to touch pad and voice input ability propose that interactions 
with natural display in innovative days. To upgrade user skills intelligent interfaces help to get information from input and output of content and direction finding for route among applications. A type of such collaboration is that point up significant interaction and lessens the user efforts to discover needed information. Similarly intelligent interfaces can adjust to give access to maximum significant utility and information [19]. Pervasive Computing is attempting to embed the innovation into nature so to make it more natural and invisible in the meantime. Pervasive displays are getting more natural day by day. Speech enables interfaces validate the utilization of natural language associate with user and devices that outwardly chase user's developments and react likewise [20]-[22]. The pervasive applications would be different from the desktop model of computing environment and interaction should be in natural style. Human like to interact with their physical environment and human's natural actions would be the root of interaction through system. Despite the fact that a considerable measure of examination with the strokes of pen and gesture input presented however the amount of natural error quiet high and ought to be settled down in future. Capacity of these natural interfaces have to gain from their environment would be not difficult to utilize. Similarly they can help user task instead of occupying the user from their task to emphasis on innovative emerges [23] [24].

\section{Pervasive Computing Security Challenges}

Pervasive Computing has more prominent convince in diverse domains on both local and worldwide situations. It is critical for analysts to recognize the challenges, objectives, and methods for mounting these technologies in diverse areas to completely aware of its potential. Pervasive Computing would detriment the entire society and absent the limits in computing. In general pervasive technology advancements will be oppressed through an advanced situation that is mindful of their presence. Natural interaction is pervasively available by means of adaptive, sensitive and receptive to their needs, habits and feelings. Progressively, a significant number of the chips around us will sense their surroundings in simple however effective ways [25]. Pervasive Computing, part of procedures and challenges need to be tended to in order to adequately make smart spaces and accomplish miniaturization. Tremendous development and examination commitments are going on towards Mark Weiser's vision [2] on adding to a framework that can sense, compute and interconnect in a manner that can make human life simple with brilliant smart objects supporting from around his surroundings. In this procedure of emergence towards a smart environment, the actual challenges to contemplate upon are the performance issues, information administration, programming support, energy efficient, trust, security and privacy of the processing device to be designed [26]-[31]. The name alone infers pervasive systems everywhere, yet with the goal achievement should be attained to; they must break up out of focus. To do this, Pervasive Computing systems must overcome following challenges. Security outline must consider standards of time and area though Pervasive Computing is expanded in various environments transparently [32]. Protection from Unauthenticated user (security), avoidance of access by an attacker through unverified techniques (integrity), giving availability to user totally (accessibility) and evading an entity from denying previous activities (non-denial) are essential factors the security model. Recognizing kind of exchanging information, conceivable distortion or misuse, shortcomings and features, the security issues in remote system base for network infrastructure can be represented [7].

- Unauthorized access;

- Viruses attack to destroy security system;

- Undefined security solutions;

- Information hacking by hackers;

- Not have system administrator;

- Weak links;

- Weak infrastructure of application;

- Weak synchronization.

Although the distributed security mechanism focused on technical ability in the side of clients, a few circumsstances require more security to address and Pervasive Computing enroll security in different methodologies (see Figure 5).

\subsection{Privacy and Trust Issues}

There is an incredible threat to the user's privacy when more applications interact with the user. The acknowledgment of context-aware Pervasive Computing angers existing privacy concerns [33]. Taking care of individual 


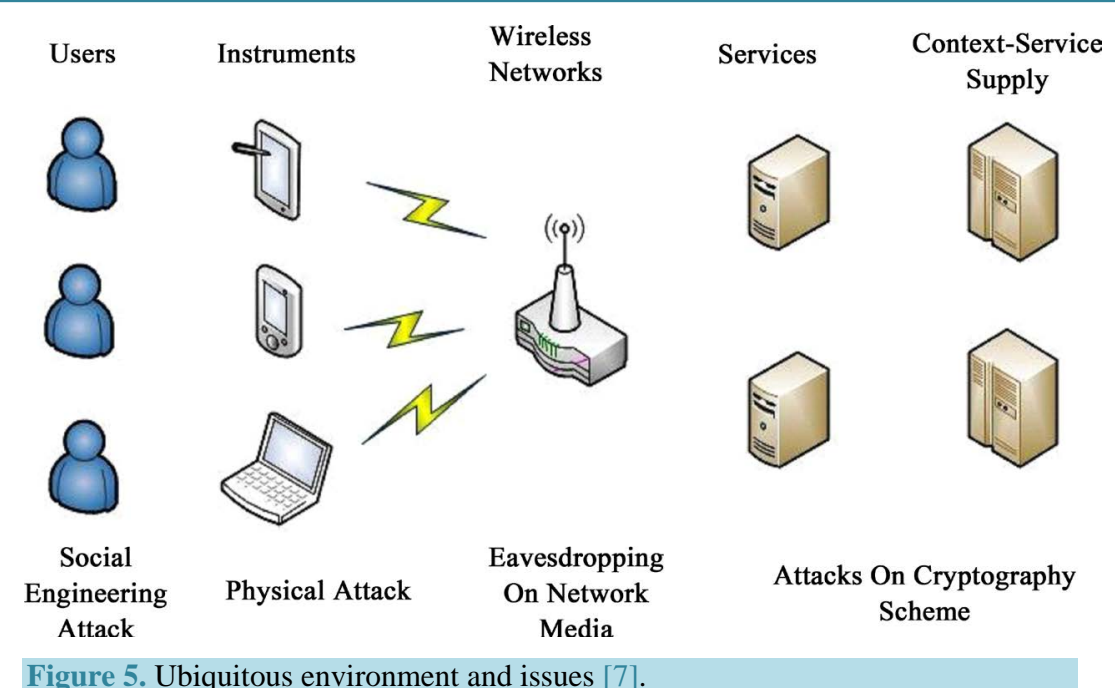

privacy is a key test in deploying Pervasive Computing administrations. The key challenge is to give a structure capable for adjusting everyday life situation and give privacy to every single user in an exceptionally dynamic pervasive environment [34]. Pervasive Computing gives ambient administrations to applications that permit users and devices in distinctive physical locations to communication naturally. It provides an environment in which devices are connected with each other and provide the facility to communicate information with least possible human participation. Since pervasive elements are always showing signs of change, trust determination is not just a static and simple methodology. The trust is the substance that holds certainty and dependence on the integrity and reliability quality of another. Trust also is a symbol of reliable, secure and trustworthy in any interaction between nodes [35].

\subsection{Social and User Interaction Issues}

Pervasive Computing is now being creates new ways of communicate, interaction, technology and considered also human behavior. Social issues are classified to recognizing what other people think and conversing with other people influenced by the system (or dependable of it), and the general social pressures of having a place with a group can all influence people impression of innovation. On account of the way of group interaction between user in the space, it is not effectively conceivable to prevent seeing or got notification from claiming user information, thus consideration to overcome because of this issue must be taken into security arrange by jointing physical and virtual parts of access control with one another [7].

\section{Solutions for Pervasive Computing Challenges}

Security plays an important role in field of Pervasive Computing environment. There are some security solutions to face pervasive systems are proposed.

\subsection{Role Based Access Control}

Role Based Access Control (RBAC) is in view of different roles on an individual happening as a component of an association. Role based access control is achieved the user role and their privileges. In this technique, every role is assigned to a set of authorization to hold a place as an order among different entities. It incorporates two sorts of mappings, which are user role assignment (URA) and role permission assignment (RPA). These are upgraded independently [7].

\subsection{Single Sing on (SSO)}

User frequently needs to get to different administration services getting included with various verification and different devices, services and networks. So it is obliged to execute a single sign-on solution which changes the initiation for entries to validate once in all system domains to incorporate reliable leaving and joining of perva- 
sive systems without disturbances [36] [37].

\subsection{Digital Right Management (DRM)}

While huge ability of conveying numerous administrations by Pervasive Computing to users is perceived, affirmation of being secure for providers in computerized environment is ensured utilizing a Digital Right Management (DRM) framework to implement in pervasive devices[36] [37].

\subsection{Ubiquitous Device Management UDM}

In dynamic environment, outgoing access effect the networks entirely, so UDM is concerned to provide the act against modification of environment to preserve availability [7].

\subsection{Radio Frequency Identification (RFID)}

The suggested methodology receives a useful acknowledgment of a protected detached (battery-less) RFID label and integrates the ultra-low power Advanced Encryption Standard (AES) design together with a novel random number generator. The test results demonstrate that the suggested comprehensive methodology can give a solid security ensure with low power, low number of cycles, and low range for accomplishing to obliged execution inside the strict imperatives forced by submissive RFIDs. RFID has traceability facility to read, transform validity and match RFID-tag to the database server for authentication [38].

\subsection{Trust Based Security Solution}

This proposal increase a security policy and assigning identification to entities. The solution has thought on extending out of SPKI and RBAC for accessibility of smart devices associated together i.e. utilizing Bluetooth. Trust base security authenticates to new user and take feedback from trusted entities is connected [7].

\subsection{Service-Oriented and User-Centric Intrusion Detection System (SUIDS)}

Intrusion detection system (IDS), have weaknesses prompting to tough deployment because of absence of consideration about heterogeneity, adaptability and resource limitation of Pervasive Computing system networks. To make sense of this issue service-oriented and user-centric intrusion detection system (SUIDS) is recommended which record events and logs to infer protection techniques on different systems devices against intrusions [7]. It joins the exceptional needs of pervasive system by selecting the restricted resource limit of administration nodes and high portability of user nodes into record. SUIDS attains to better execution regarding energy efficiency whereas having high detection efficiency. The normal lifetime of node is expanded $23.9 \%$ and the lifetime of network is expanded $17.8 \%$ [39].

\subsection{Biometrics}

Biometric system is basic to first examine the information and consolidate this understanding inside the recognition system and making evaluation of biometric quality an imperative part of biometrics. Biometric system experience variability in information that impact capture, treatment, and usage of a biometric example. It offers consistent and computerized systems to deciding and affirming identity. Finger print, face and iris recognition techniques are rapidly incoming as a secure source of passwords by reading image feature techniques [40].

\section{Conclusion}

Technology is rapidly finding its method and changing states faster than speed into every aspect of our lives as a basic need of time. The way of the pervasive environment permits communication between devices whenever and everywhere, so the systems become more pervasive in modern world. Pervasive Computing will be a ripe resource of challenging research issues in computer systems for a long time to come. We will need to address study and explore the challenges in areas outside computer systems. Interaction abilities will need to be emergence with the sorts of computer systems functionalities and answering different applications appearances will oblige us to expand our topic on a few ideas. The role of Pervasive Computing from these areas will need to be integrated into existing physical environment and newly built structures discuss in this paper. Existing innova- 
tive abilities give the potential to satisfy Weiser's vision. What has been required is the expression of ideas that will empower designers to deliberately design for physical and cognitive accessibility. Pervasive Computing gives an appealing vision to the future of computing systems. Security, trust and privacy design methods are getting importance in HCI with the advancement of flexibility, nontraditional computing applications that have a solid effect on the personal, natural and instructive privacy of users. To develop security models we recommend to elegant designers by helping they see better work that goes into everyday security, trust and privacy. Hence, research in this field should build familiarity with the impacts of applying specific methods, and should help selecting whatever design methodology is most proper for the configuration of current workload. To accomplish this objective; we propose to develop the security methodologies. A number of the challenges we showing are sensible, applicable and within reach, making them prime challengers for rich future advancements.

\section{Acknowledgements}

Authors are grateful to respectable Prof. Syed Ghulam Qasim Shah who reviewed our research work.

\section{References}

[1] Bashir, R.N., Qadri, S., Saleem, R.M., Naeem, M. and Ghafoor, Y. (2014) Human Computer Interaction (HCI) in Ubiquitous Computing. International Journal of Innovation and Applied Studies, 9, 534.

[2] Weiser, M. (1991) The Computer for the 21st Century. Scientific American, 265, 94-104. http://dx.doi.org/10.1038/scientificamerican0991-94

[3] Fouad, R., Hashem, M., Badr, N. and Talha, H. (2011) Exploring a Hybrid of Geospatial Semantic Information in Ubiquitous Computing Environments. Global Journal of Computer Science and Technology, 11(19).

[4] Brodersen, C. and Kristensen, J.F. (2004) Interaction through Negotiation. Proceedings of the 3rd Nordic Conference on Human-Computer Interaction, 259-268. http://dx.doi.org/10.1145/1028014.1028054

[5] Sandhu, R. (2013) Shifting Paradigm from Mobile Computing to Ubiquitous/Pervasive Computing. Indexing Journal Indexing: Our Journal Has Recently Joined International Database for Indexing with DOAJ, Index Copernicus, Open J Gate, CAS, Google Scholar.

[6] (2013) Pervasive Computing. IEEE Computer Society, 12, 18-20.

[7] Sharifi, A. and Abdulahshah, M.K. (2013). Security Attacks and Solutions on Ubiquitous Computing Network. International Journal of Engineering and Innovative Technology (IJEIT), 3, 40-45.

[8] Ye, J. and Dobson, S. Pervasive Computing Needs Better Situation-Awareness. Awareness Magazine. http://dx.doi.org/10.2417/3201201.003943

[9] Ismail, A., Hajjar, A.E.S.A. and Ismail, Z. (2011) A New System Architecture for Pervasive Computing. arXiv Preprint arXiv:1108.2389.

[10] Abowd, G.D. and Mynatt, E.D. (2000) Charting Past, Present, and Future Research in Ubiquitous Computing. ACM Transactions on Computer-Human Interaction (TOCHI), 7, 29-58. http://dx.doi.org/10.1145/344949.344988

[11] Joshi, Y. and Prasad, L. (2012) Pervasive Computing Goals and Its Challenges for Modern Era. International Journal of Computer Science and Network (IJCSN), Volume 1, Issue 3, ISSN 2277-5420. www.ijcsn.org

[12] Nielsen, J. (1993) Noncommand User Interfaces. Communications of the ACM, 36, 83-99. http://dx.doi.org/10.1145/255950.153582

[13] Morariu, A., Clipa, B.D., De Lausnay, S., De Strycker, L. and Pentiuc, S. (2011) Combining Natural Human-Computer Interaction and Wireless Communication. Journal of Applied Computer Science \& Mathematics, 5, 47-52.

[14] Schmidt, A. (2013) Context-Aware Computing: Context-Awareness, Context-Aware User Interfaces, and Implicit Interaction. In: Soegaard, M. and Dam, R.F., Eds., The Encyclopedia of Human-Computer Interaction, 2nd Edition, The Interaction Design Foundation, Aarhus.

[15] Chihani, B., Bertin, E. and Crespi, N. (2011) A Comprehensive Framework for Context-Aware Communication Services. Proceedings of the 15th International Conference on Intelligence in Next Generation Networks (ICIN), Berlin, 4-7 October 2011, 52-57. http://dx.doi.org/10.1109/icin.2011.6081102

[16] Chihani, B., Bertin, E., Jeanne, F. and Crespi, N. (2011) Context-Aware Systems: A Case Study. In: Cherifi, H., Zain, J.M. and El-Qawasmeh, E., Eds., Digital Information and Communication Technology and Its Applications, Springer, Berlin, 718-732. http://dx.doi.org/10.1007/978-3-642-22027-2_60

[17] Karray, F., Alemzadeh, M., Saleh, J.A. and Arab, M.N. (2008) Human-Computer Interaction: Overview on State of the Art. International Journal on Smart Sensing and Intelligent Systems. 
[18] Hachman, M. (2002) Canesta Says “Virtual Keyboard” Is Reality. http://www.extremetech.com/extreme/51958-canesta-says-virtual-keyboard-is-reality

[19] Grudin, J. (2011) A Moving Target: The Evolution of HCI. In: Jacko, J., Ed., The Human-Computer Interaction Handbook, 3rd Edition, Taylor \& Francis, New York.

[20] Oviatt, S., Cohen, P., Wu, L., Duncan, L., Suhm, B., Bers, J., et al. (2000) Designing the User Interface for Multimodal Speech and Pen-Based Gesture Applications: State-of-the-Art Systems and Future Research Directions. Human-Computer Interaction, 15, 263-322. http://dx.doi.org/10.1207/S15327051HCI1504 1

[21] Gavrila, D.M. (1999) The Visual Analysis of Human Movement: A Survey. Computer Vision and Image Understanding, 73, 82-98. http://dx.doi.org/10.1006/cviu.1998.0716

[22] Sibert, L.E. and Jacob, R.J. (2000) Evaluation of Eye Gaze Interaction. Proceedings of the SIGCHI Conference on Human Factors in Computing Systems, The Hague, 1-6 April 2000, 281-288.

[23] Zacharia, K., Elias, E.P. and Varghese, S.M. (2011) Modelling Gesture Based Ubiquitous Applications. http://arxiv.org/abs/1112.2044

[24] Poslad, S. (2011) Ubiquitous Computing: Smart Devices, Environments and Interactions. John Wiley \& Sons, Chichester.

[25] Chandini, N., Reddy, N.C.S. and Bashwanth, N. (2014) Pervasive Computing Goals and Its Challenges for New Epoch. International Journal of Advanced Research in Computer and Communication Engineering, 3, 6437-6439.

[26] Rao, D.H. (2014) A Scenario Based Approach for Dealing with Challenges in a Pervasive Computing Environment. http://arxiv.org/ftp/arxiv/papers/1405/1405.6661.pdf

[27] Das, S.K., Kant, K. and Zhang, N. (2012) Handbook on Securing Cyber-Physical Critical Infrastructure. Elsevier, Amsterdam.

[28] Roy, N., Misra, A., Julien, C., Das, S.K. and Biswas, J. (2011) An Energy-Efficient Quality Adaptive Framework for Multi-Modal Sensor Context Recognition. Proceedings of the IEEE International Conference on Pervasive Computing and Communications (PerCom), Seattle, 21-25 March 2011, 63-73. http://dx.doi.org/10.1109/percom.2011.5767596

[29] Rajkumar, R. and Lee, I. (2006) NSF Workshop on Cyber-Physical Systems. 16-17 October 2006, Austin. http://varma.ece.cmu.edu/CPS/

[30] Hayes, G.R., Poole, E.S., Iachello, G., Patel, S.N., Grimes, M., Abowd, G.D. and Truong, K.N. (2007) Physical, Social, and Experiential Knowledge in Pervasive Computing Environments. IEEE Pervasive Computing, 6, 56-63. http://dx.doi.org/10.1109/MPRV.2007.82

[31] Campbell, A.T., Eisenman, S.B., Lane, N.D., Miluzzo, E., Peterson, R., Lu, H., et al. (2008) The Rise of People-Centric Sensing. IEEE Internet Computing, 12, 12-21. http://dx.doi.org/10.1109/MIC.2008.90

[32] Leung, A., Sheng, Y. and Cruickshank, H. (2007) The Security Challenges for Mobile Ubiquitous Services. Information Security Technical Report, 12, 162-171. http://dx.doi.org/10.1016/j.istr.2007.05.001

[33] Pallapa, G., Kumar, M. and Das, S.K. (2007) Privacy Infusion in Ubiquitous Computing. Proceedings of the Fourth Annual International Conference on Mobile and Ubiquitous Systems: Networking \& Services, Philadelphia, 6-10 August, 1-8. http://dx.doi.org/10.1109/mobiq.2007.4451030

[34] Ganesh, M. and Krishna, S.M. (2010) Privacy Enhanced Context-Aware Architecture for Ubiquitous Computing. International Journal of Electronics and Computer Science Engineering, 2, 53-64.

[35] Khiabani, H., Sidek, Z.M. and Manan, J.L.A. (2010) Towards a Unified Trust Model in Pervasive Systems. Proceedings of the 24th International Conference on Advanced Information Networking and Applications Workshops (WAINA), Perth, 20-23 April 2010, 831-835. http://dx.doi.org/10.1109/waina.2010.144

[36] Campbell, R., Al-Muhtadi, J., Naldurg, P., Sampemane, G. and Mickunas, M.D. (2003) Towards Security and Privacy for Pervasive Computing. In: Okada, M., Pierce, B.C., Scedrov, A., Tokuda, H. and Yonezawa, A., Eds., Software Security—Theories and Systems, Springer, Berlin, 1-15. http://dx.doi.org/10.1007/3-540-36532-X_1

[37] Forné, J., Hinarejos, F., Marín, A., Almenárez, F., Lopez, J., Montenegro, J.A., et al. (2010) Pervasive Authentication and Authorization Infrastructures for Mobile Users. Computers \& Security, 29, 501-514. http://dx.doi.org/10.1016/j.cose.2009.09.001

[38] Wang, G., Zhou, W. and Yang, L.T. (2013) Trust, Security and Privacy for Pervasive Applications. The Journal of Supercomputing, 64, 661-663. http://dx.doi.org/10.1007/s11227-013-0953-4

[39] Zhou, B., Shi, Q. and Merabti, M. (2007) Towards Energy-Efficient Intrusion Detection in Pervasive Computing. Proceedings of the IEEE International Conference on Communications, Glasgow, 24-28 June 2007, 1417-1422. http://dx.doi.org/10.1109/icc.2007.238

[40] Bharadwaj, S., Vatsa, M. and Singh, R. (2014) Biometric Quality: A Review of Fingerprint, Iris, and Face. EURASIP Journal on Image and Video Processing, 2014, 34. http://dx.doi.org/10.1186/1687-5281-2014-34 